\title{
Longitudinal volumetric MRI study of pituitary gland following severe traumatic brain injury
}

\author{
Sorin C. Craciunas ${ }^{1, \star}$, Carmen M. Cirstea ${ }^{1,2,4}$, Hung-Wen Yeh ${ }^{3}$, \\ Lewis Hutfles ${ }^{1}$, JoAnn Lierman ${ }^{1}$, Allan Schmitt ${ }^{1}$, William M. Brooks ${ }^{1,4}$ \\ ${ }^{1}$ Hoglund Brain Imaging Center; Departments of ${ }^{2}$ Physical Therapy \& \\ Rehabilitation Science, ${ }^{3}$ Biostatistics and ${ }^{4}$ Neurology, University of Kansas Medical \\ Center, Kansas City, Kansas, USA *Current address: Neurosurgery Unit IV, \\ Bagdasar - Arseni Hospital, Bucharest, Romania
}

\begin{abstract}
Purpose: Previous studies have suggested that the hypopituitarism following traumatic brain injury (TBI) is more prevalent than traditionally thought. The objective of this study was to characterize longitudinal MRI morphometric changes of pituitary gland in patients with severe TBI.

Materials and methods: Fourteen patients who had suffered a severe TBI (Glasgow Coma Score $=3$ - 8) underwent MRI at three time points: Time 1 (mean $=31.5$ days), Time 2 (98. 0) and Time 3 (185. 5). The pituitary gland volume was quantified by manually tracing on T1-weighted magnetic resonance images. Data from TBI patients were compared to 14 age- and sexmatched uninjured controls. The relationships between pituitary volumetric measures and patient demographics, length of respiratory support and coma, and presence of intracranial hemorrhage or skull fractures were also analyzed.

Results: Following TBI, the pituitary volumes were significantly greater at all three time points: Time 1: median $=665 \mathrm{~mm} 3, \quad$ range $=460-830 \mathrm{~mm} 3$, $\mathrm{p}=0.007$; Time 2: 694mm3, 467-866mm3, 0 . 007; and Time 3: 655mm3, 444$795 \mathrm{~mm} 3$, 0. 015, compared with controls
\end{abstract}

(504mm3, 433-591mm3). At Time 1, pituitary volume was increased in 10 out of 14 patients. Of these, early pituitary enlargements persisted up to six months in nine patients. Pituitary enlargements were negatively correlated with GCS, but not with other variables.

Conclusion: Following a severe TBI, early pituitary enlargement found in most of our patients persisted in the chronic phases. Our data suggest a potential role of MRI morphometry in early prediction of pituitary dysfunction following head trauma, but further studies including hormonal measurements are necessary for validation.

Key words: neuroimaging, traumatic brain injury, hypopituitarism

\section{Introduction}

Traumatic brain injury (TBI) represents a major cause of mortality and long-term morbidity. In the United States alone, each year approximately 1. 4 million people sustain a TBI(17) with an estimated cost of $\$ 60$ billion. (10) At present, there are at least 5.3 million TBI survivors, about $2 \%$ of the U. S. population, with long-term disabilities. (33) 
Although pituitary damage following TBI was reported as early as 1918,(7) interest in this clinical entity has grown with recent studies consistently reporting a high-risk of hypopituitarism, determined by endocrine testing, with an incidence of $30-70 \%$ following TBI. (1-3, 5, 12, 18, 28) Despite this incidence, pituitary function testing has not become routine for patient management after TBI. Since the pituitary gland has the role of "endocrine brain", we could speculate that the failure to diagnose post head trauma hypopituitarism (PHTH) might contribute considerably to morbidity and mortality in TBI patients. Consequently, the signs and symptoms of PHTH might be overlooked and could be interpreted as being post-TBI cognitive/behavioral sequelae. For example, clinical complaints such as headache, irritability, poor concentration and memory, depression, fatigue, decrease in libido, although considered symptoms of postconcussional syndrome, can also result from hypopituitarism, particularly of growth hormone (GH) deficiency. (9, 19, 21, 24) Hormonal substitution therapies are used successfully for non-traumatic hypopituitarism and it has been suggested that TBI-mediated hypopituitarism might also benefit from appropriate hormonal supplements. $(9,21)$

Numerous attempts have been made to define criteria or to identify high-risk factors that indicate the need for endocrine testing post head trauma, $(1,3,4,13,14,18$, $24,26,28,30,31)$ but no valid prognostic index has been found to be sufficiently accurate. Since imaging investigations (CT, $\mathrm{MRI}$ ) are mandatory in the management of most TBI patients, the identification of a quantitative neuroimaging biomarker of PHTH might be a reliable and cost- effective tool to determine which TBI patients require endocrine testing and follow-up.

The major goal of the present study was to quantify pituitary volume changes in the first six months after TBI. The relationship between pituitary volume changes, measured from MRI, and clinical factors of TBI severity (e. g. , skull fractures, length of coma) was also studied. We focused on severe TBI, since this group has a high-risk for developing PHTH, and on the first six months post-TBI when pituitary hormonal alterations are dynamic. $(11,15,23,30,31)$

\section{Materials and Methods}

\section{Participants}

Fourteen TBI patients ( 4 women; age median $=23 \mathrm{yrs}, \quad$ range $=16-47 \mathrm{yrs}$ ) participated. Inclusion criteria were: severe TBI (Glasgow Coma Score, GCS $<8$ ); age: 18-55 years; and body mass index: 17-30 $\mathrm{kg} / \mathrm{m} 2$ (see Table 1). Exclusion criteria were: previous and/or penetrating TBI; preexisting neurological and/or endocrinological disease; systemic conditions, such as chronic metabolic disturbance, hepatic failure, uremia, kidney transplant, uncontrolled diabetes; major substance abuse; and treatment with neuroleptic or antiepileptic drugs prior to the event. The severity of TBI was based on GCS assessment at 24 hours post-TBI(32) to minimize the possible effect of confounding factors that could overestimate the severity of trauma at emergency room (i.e., illicit drugs, sedation, alcohol). Histories for each patient, including the injury type, estimated severity and patient condition at presentation (from family interviews, medical chart reviews, emergency room notes, police reports) were obtained. Neurologic signs, vital 
functions, history of resuscitation, degree of skeletal and visceral injury, cranio-cerebral lesions (skull fractures, posttraumatic intracranial hemorrhage/hematoma), anesthesia, respiratory support, and coma days were also recorded.

Fourteen age- and sex-matched uninjured controls (4 women; 24. 5yrs, 18$47 y r s)$ without history of neurological and endocrinological disorder or drugs/alcohol abuse were recruited.

All participants were informed of the experimental procedures and gave their written consent to this study that was approved by the local Institutional Review Board.

\section{MRI assessment and analysis}

Magnetic resonance imaging was carried out using a 3 Tesla Siemens Allegra scanner (Siemens Medical Solutions, Erlangan, Germany). We studied TBI patients at one month, when they were clinically stable for transport to our outpatient imaging facility (Time 1: actual mean $=31.5$ days), three (Time $2=98.0$ days) and six months (Time $3=185.5$ days) post-injury. A 3D axial T1weighted series (magnetization-prepared rapid gradient echo - MPRAGE, $\mathrm{TR}=1520 \mathrm{~ms}, \quad \mathrm{TE}=4 . \quad 38 \mathrm{~ms}, \mathrm{TI}=900 \mathrm{~ms}$, flip angle $=8$ degree, $256 \times 156 \mathrm{~mm} 2$ matrix, 1. $5 \mathrm{~mm}$ slice thickness) was collected. We paid careful attention to patient positioning at each time point by using the reference landmarks as described previously. (6)

Anterior and posterior pituitary gland was outlined manually on the T1-weighted images and the volume quantified by multiplying the traced area in each slice by the slice thickness and summing over all slices (MedInria v2. 0. Software, INRIA, Sofia Antipolis, France). The pituitary boundaries were drawn on the axial slices (Figure 1A and B), with confirmation on the simultaneously displayed sagittal and coronal planes (3D reconstruction, Figure $1 \mathrm{C}$ and $\mathrm{D})$, by a neurosurgeon. MRI data were analyzed blinded to group and time point.

\section{Accuracy and Reproducibility of Pituitary Volume Measurements}

To verify the anatomical accuracy of tracing, one investigator traced the pituitary boundaries on six randomly selected image sets. Then, a second investigator compared the tracings with an anatomic atlas (22). In the case of inaccuracy the tracing was reexecuted. This cycle was repeated until agreement was reached.

To determine intra-rater reliability, 10 pituitary image sets were traced by one investigator on two different occasions separated by 7-10 days. The second tracing was executed without reference to the first.

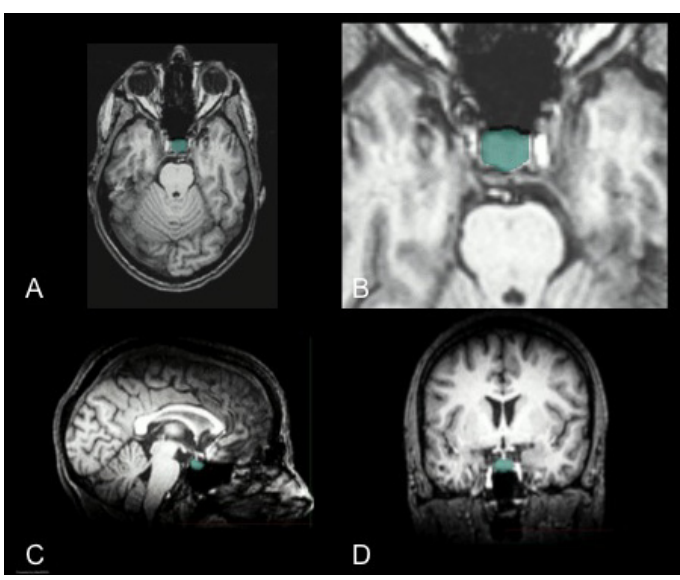

Figure 1 Measurement of pituitary gland volume. (A) Outline of pituitary gland shown on complete axial T1-weighted slice $(\mathrm{TR}=1520 \mathrm{~ms}, \mathrm{TE}=4.38 \mathrm{~ms}$, $\mathrm{TI}=900 \mathrm{~ms}$, flip angle $=8$ degree). (B) Magnified view of A showing detailed anatomy. (C) and (D) Corresponding sagittal and coronal views that are viewed simultaneously while tracing to ensure accurate boundaries

The intra-rater reproducibility was extremely high (Spearman coefficient $r=1$. $00 ; \mathrm{p}<0.0001)$. 
To determine inter-rater reliability, one investigator traced the pituitary boundaries on eight randomly selected image sets. A third investigator then traced these pituitary image sets without reference to the results of the previous investigator. The reliability between these independent raters was high (Spearman $\mathrm{r}=0.81 ; \mathrm{p}<0.02$ ).

\section{Statistical analysis}

Since the sample size was small, we used nonparametric methods obviating the need for assumptions of normal distributions in pituitary volume measurements. Analysis focused on one outcome measure (pituitary volume) and five variables (GCS at 24 hours; number of days on ventilator support; number of days of coma; intracranial hemorrhagic lesions, where $1=$ present and $0=$ absent; and skull fractures, where $1=$ present and $0=$ absent).

The between-group comparisons at each time point were conducted by the Wilcoxon rank-sum/Mann-Whitney $U$ test whereas the within group (TBI) comparisons were conducted by the Wilcoxon signed-rank test.

To address the question of whether presence of overt injury was associated with pituitary volumes, we divided the TBI group based on the presence of either hemorrhage or skull fracture. We then used the Wilcoxon Rank-sum test to determine whether pituitary volumes were different for any subgroup at any time point.

The relationships between pituitary volumes and clinical injury severity variables were estimated by the Spearman's correlation coefficients between the pituitary volume at each time point and each of the five variables.

Finally, we divided the TBI group according to their pituitary volume at Time
1. We considered any pituitary volume that fell within the normal range of our healthy group mean to be normal and volumes that fell outside this range as abnormal. We then repeated the aforementioned analyses for the abnormal and normal TBI subgroups separately.

All hypothesis testing was 2-sided and we did not attempt to control the experiment-wise Type I error rate. The significance level was set at $\alpha=0.05$.

\section{Results}

\section{Patient characteristics}

The causes of injury included motor vehicle accident $(n=10)$, fall $(2)$, and assault (2) (see Table 1), resulting in a severe TBI (GCS at 24 hours ranged from three to seven; seven patients had a GCS of three) with length of coma ranging between two and eight days (median=3. 5 days). All patients received oro-tracheal intubation and ventilator support (median $=5.5$ days, range $=2-19$ days). Associated intracranial hemorrhagic lesions were present in $71 \%$ $(10 / 14)$ of patients and fractures of skull vault in $43 \%(6 / 14)$. No patient had a skull base fracture. Half of the patients $(n=7)$ presented with multiple trauma, including hemo- and/or pneumothorax (six patients), fractures (vertebral, pelvis, extremities), spleen laceration, and kidney contusion.

\section{Pituitary volume measurements}

The pituitary volume measurements were summarized by medians and ranges for each group and each time point (Table 2). Compared with uninjured controls, the pituitary volumes in TBI survivors were significantly greater at all time points (see Table 2 and Figure 2). 
DOI: 10.2478/v10282-012-0009-y

TABLE 1

Demographic and clinical data of TBI survivors

\begin{tabular}{|c|c|c|c|c|c|c|c|c|}
\hline Patient & $\begin{array}{l}\text { Age/ } \\
\text { Sex }\end{array}$ & BMI & $\begin{array}{l}\text { TBI } \\
\text { cause }\end{array}$ & $\begin{array}{l}\text { GCS } \\
(24 \mathrm{hr})\end{array}$ & $\begin{array}{l}\text { Intracranial } \\
\text { hemorrhage }\end{array}$ & $\begin{array}{c}\text { Skull } \\
\text { fracture }\end{array}$ & $\begin{array}{l}\text { Coma } \\
\text { length } \\
\text { (days) }\end{array}$ & $\begin{array}{c}\text { Respirator } \\
\text { support } \\
\text { (days) }\end{array}$ \\
\hline 1 & $41 / \mathrm{M}$ & 23.6 & MVA & 3 & & & 5 & 6 \\
\hline 2 & $16 / \mathrm{M}$ & 23.3 & MVA & 3 & + & + & 6 & 11 \\
\hline 3 & $47 / M$ & 21.5 & Assault & 3 & + & + & 8 & 10 \\
\hline 4 & $24 / \mathrm{M}$ & 22.8 & Fall & 3 & + & & 2 & 4 \\
\hline 5 & $20 / \mathrm{F}$ & 19.9 & MVA & 3 & & & 3 & 3 \\
\hline 6 & $21 / \mathrm{F}$ & 25.0 & MVA & 3 & + & & 7 & 15 \\
\hline 7 & $18 / \mathrm{M}$ & 21.5 & MVA & 3 & + & + & 3 & 5 \\
\hline 8 & $27 / \mathrm{F}$ & 28.8 & MVA & 5 & + & + & 1 & 2 \\
\hline 9 & $24 / M$ & 22.2 & MVA & 6 & & & 3 & 14 \\
\hline 10 & $22 / \mathrm{M}$ & 19.2 & MVA & 6 & + & + & 5 & 5 \\
\hline 11 & $21 / \mathrm{M}$ & 23.0 & MVA & 6 & + & & 3 & 7 \\
\hline 12 & $47 / \mathrm{F}$ & 22.0 & Assault & 6 & & + & 2 & 2 \\
\hline 13 & $26 / \mathrm{M}$ & 25.4 & MVA & 7 & + & & 6 & 19 \\
\hline 14 & $22 / \mathrm{M}$ & 22.1 & Fall & 7 & + & & 4 & 4 \\
\hline
\end{tabular}

Note. BMI = Body Mass Index; $\mathrm{F}=$ female; GCS = Glasgow Coma Score; $\mathrm{M}=$ male; $\mathrm{MVA}=$ motor vehicle accident; $+=$ present. Patients are ranked according to Glasgow Coma Score.

Overall, these values suggest that following TBI, pituitary volumes are higher than normal (uninjured control vs. Time 1; $\mathrm{p}=0.007)$, followed by further enlargement up to three months (Time 2 vs. $1 ; \mathrm{p}=0.09$ ) and finally apparent return toward normal range at six months post TBI (Time 3 vs. 2; $\mathrm{p}=0.02$; Table 2 and Figure 2).

At Time 1, 10 of the TBI patients (71\%; TBI patients \# 3-7 and 9-13 in the Table 2) had pituitary volumes outside the normal range of the uninjured group. In this TBI subgroup (abnormal TBI subgroup), the median pituitary volume increased slightly from 718 to $753 \mathrm{~mm} 3$ at Time 2 (Time 2 vs. $1, p=0.06)$ and decreased to $673 \mathrm{~mm} 3$ at Time 3 (Time 3 vs. 2 and $1, \mathrm{p}=0.01$ and 0 . 16 respectively). Although the pituitary volumes decreased between Times 2 and 3, the volumes measured at Time 3, nonetheless, remained above the uninjured group range in nine of the patients. 
TABLE 2

Pituitary volumes (mm3) in TBI survivors and healthy controls

\begin{tabular}{|c|c|c|c|c|}
\hline \multirow[t]{2}{*}{ Subject } & \multicolumn{3}{|c|}{ TBI patients } & $\begin{array}{l}\text { Uninjured } \\
\text { controls }\end{array}$ \\
\hline & Time 1 & Time 2 & Time 3 & \\
\hline 1 & 499 & 513 & 507 & 433 \\
\hline 2 & 460 & 481 & 486 & 501 \\
\hline 3 & 739 & 866 & 659 & 499 \\
\hline 4 & 711 & 760 & 743 & 571 \\
\hline 5 & 830 & 811 & 795 & 504 \\
\hline 6 & 794 & 781 & 740 & 548 \\
\hline 7 & 761 & 820 & 659 & 529 \\
\hline 8 & 584 & 528 & 504 & 494 \\
\hline 9 & 725 & 706 & 550 & 456 \\
\hline 10 & 645 & 654 & 617 & 492 \\
\hline 11 & 662 & 683 & 651 & 512 \\
\hline 12 & 668 & 747 & 688 & 504 \\
\hline 13 & 615 & 666 & 693 & 576 \\
\hline 14 & 470 & 467 & 444 & 591 \\
\hline Median & $665^{\ddagger}$ & $694^{\ddagger}$ & $655^{\dagger}$ & 504 \\
\hline Range & $460-830$ & $467-866$ & $444-795$ & $5456-591$ \\
\hline Mean \pm SD & $654 \pm 118$ & $677 \pm 133$ & $624 \pm 109$ & $9 \quad 521 \pm 45$ \\
\hline
\end{tabular}

Note. $† \mathrm{p}<0.05 ; \ddagger \mathrm{p}<0.01$ compared with uninjured controls. Patients 1, 2, 8, and 14 remained within the normal range throughout the study period.

The four patients (\# 1, 2, 8, and 14) with pituitary volumes within the normal range at Time 1 (normal TBI subgroup) did not show significant progression over time (median $=465,497$, and $485 \mathrm{~mm} 3$ at Times 1,2 , and 3 , respectively).

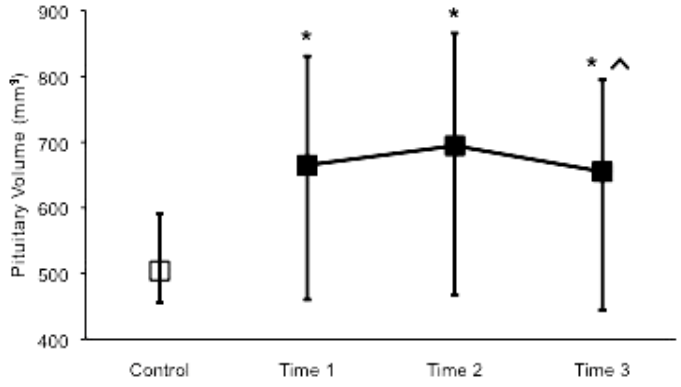

Figure 2 Median and range pituitary volumes $(\mathrm{mm} 3)$ in uninjured (open square) and TBI (filled square) groups. In the TBI group, pituitary volumes were measured at three time points: Time 1 (one month-post TBI), Time 2 (three months) and Time 3 (six months). Asterisks ( $\left.{ }^{\star}\right)$ indicate significant differences between TBI and healthy groups, and carat $\left({ }^{\wedge}\right)$ between Time 3 and Time 2 in TBI group $(\mathrm{p}<0.05)$

\section{Correlations between pituitary volume} measurements and clinical variables

Although, for the entire TBI group, the pituitary volume at all time points was negatively correlated with the GCS, none of these correlations was significant $(\mathrm{p}=0.16$, 0.10 , and 0.33 at Times 1,2 , and 3 respectively). However, for the abnormal TBI subgroup, we found stronger correlations at all time points (Time 1: $\mathrm{r}=$ 0. 84, $\mathrm{p}=0$. 002; Time 2: $\mathrm{r}=-0.87, \mathrm{p}=0$. 001; Time 3: $r=-0.46, p=0.18$; see Figure 3).

For both the whole TBI group and the abnormal subgroup, correlations between pituitary volume and number of coma days or ventilator support days did not reach statistical significance at any time point. No significant difference was found between the patients with and those without intracranial hemorrhagic lesions or skull fractures. 


\section{Discussion}

We found substantially larger pituitary volumes in the majority of survivors of severe TBI compared with uninjured controls. We interpret the larger volumes as indicating injury-related enlargement. Moreover, in these patients, the pituitary volume tended to increase until three months. Although there was some indication of recovery by six months postinjury, pituitary volume in all but one patient remained above the normal range. In contrast, none of the patients with pituitary volumes in the normal range at one month post-injury presented with pituitary enlargement over the same period.

Our findings are consistent with recent reports of pituitary enlargement in the acute (0-7 days) phase of moderate to severe TBI(20). However, an association between increased pituitary volume and pituitary dysfunction following TBI is still to be elucidated. The hypothalamus-pituitary axis can be directly or indirectly altered by TBI. Hypoxia, edema, intracranial hemorrhage or increased intracranial pressure,(8) subsequent to trauma of either pituitary, hypothalamus, stalk, or other brain regions are potential mechanisms underlying such dysfunction. Histological studies of TBIinduced alterations of this axis have found various degrees of pituitary hemorrhage, ischemic necrosis, fibrosis and/or thrombosis. (1, 24-26) However, the most prevalent mechanisms associated with pituitary dysfunction seem to be hemorrhage and ischemia. (13) To date, no correlations between the type or extent of histological pituitary alterations and characteristics of trauma, such as mechanism, extension and type of brain injuries, or skull fractures, have been reported,(24) although one study found no histological changes in 14 to $74 \%$ of TBI cases. (26) In addition, pituitary dysfunction might be secondary to other TBI-related factors, such as cardiac arrest and medications used post-TBI (e. g. , etomidate, heparin, anticonvulsants, benzodiazepines). $(8,24)$ Furthermore, an autoimmune process involving hypothalamic-pituitary region triggered by head trauma might play a role in the development of pituitary dysfunction post TBI. (29)

The trend toward normalization of pituitary volume at six months post-TBI was also suggested at 12 months follow-up. (20) Recently, an integrative neuroimaging/hormonal study of chronic (17 $\pm 15 \mathrm{yrs})$ TBI patients reported pituitary gland volume changes in $80 \%$ of patients with hypopituitarism, and in only $29 \%$ without,(27) with loss of volume or empty sella the most common abnormality seen. Taken with those of other studies, $(20,30)$ our data raise the question of whether the reductions of pituitary volumes seen in the chronic phases of TBI represent true recovery or whether it is a step toward further deterioration (i. e. , atrophy) as suggested by Schneider et al. (27) Further studies over an extended period might answer this question. Nevertheless, over a period of 3 years post-TBI,(31) a recovery of pituitary function was reported in most patients with worsening or new onset of hypopituitarism rarely occurring.

In our patients, the pituitary volume was correlated with TBI severity i. e., low GCS at 24 hours predicted larger volume (see Figure 3). Although our result is consistent with previous findings that GCS at the emergency room predicts the development of PHTH, (12) other studies reported no association between GCS and the prevalence of PHTH. $(3,18)$ 


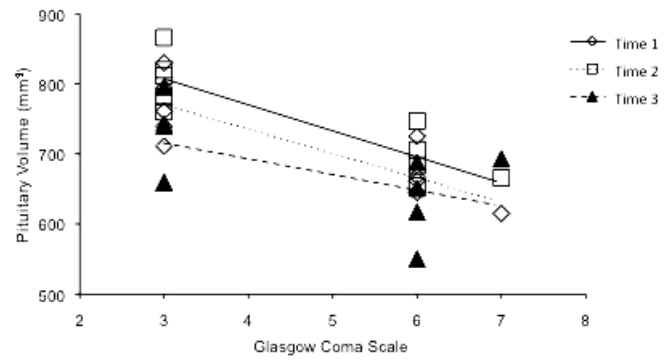

Figure 3 Correlations between pituitary volumes at one (Time 1), three (Time 2) and six (Time 3) months post-TBI and Glasgow Coma Score 24 hours post-event in the TBI abnormal subgroup $(n=10)$ that presented with significantly largely pituitary gland volume at Time 1 compared to the uninjured group.

Several risk factors associated with injury severity have been identified in the context of PHTH. One of these is presence of skull base fracture. (28) However, none of our patients presented with skull base fracture. In contrast, some of our patients sustained fractures of the cranial vault. However, the presence of cranial vault fractures was not correlated with pituitary volume changes. Another risk factor for hypopituitarism is the presence of subarachnoid hemorrhage, $(2,5,16)$ which might indicate the need for follow-up to detect delayed hypopituitarism. (19) However, we found no relationship between the presence of intracranial hemorrhagic lesions and pituitary enlargement. Finally, we found no significant correlation between pituitary enlargement and length of ventilator support at any time point.

The present study has some limitations: (i) we focused on severe TBI patients who survived for six months after the event, which might contribute a selection bias i. e., the event was serious enough to induce a severe, but not terminal, TBI; (ii) we acquired the imaging data in the axial, rather than the commonly-used coronal, plane. However, we used the same 3D reconstruction to obtain reference coronal and sagittal images in survivors and uninjured controls and do not expect bias from this source; (iii) although the small sample size might limit the generalizability of our results, this approach may be used to determine post-traumatic morphometric pituitary changes. However, in the absence of hormonal determination, any attempt to suggest the presence of hypopituitarism remains speculative. Longitudinal endocrinological studies have shown that PHTH occurs mainly in the acute phase of TBI, but later appearance has been also described. Giordano et al. (11) reported that pituitary insufficiency at three months postTBI persisted at 12 months follow-up in patients with multiple hormonal dysfunctions, but recovered in those with only isolated hormonal dysfunction. Additionally, $5 \%$ of cases with initially normal pituitary function presented with subsequent pituitary insufficiency. This is further confirmed by the recently reported appearance of new PHTH cases at 12 and 36 months post-TBI. (30), (31) In contrast, a prospective study (15) suggests that recovery of PHTH is the sole tendency in TBI patients at 12 months, with no new insufficiencies reported during that time period. Thus, the functional (hormonal) profile of the pituitary gland seems to be quite dynamic following TBI, and a combined, imaging and hormonal, approach might increase the understanding of the mechanisms underlying hypopituitarism following head trauma. Since the sub-acute stage (one month post) represents a potentially rich set of restorative therapy targets, these results would be especially useful for prospectively predict patient risk for developing 
DOI: $10.2478 / \mathrm{v} 10282-012-0009-y$

hypopituitarism. Our results might help clinical decision-making and determining individual treatment strategies.

\section{Summary Statement/Conclusions}

Larger pituitary volumes were found at one month after injury in the majority of survivors of severe TBI compared with uninjured controls, with elevated, or normal, pituitary volumes at three or six months predicted by the volumes observed at one month after injury.

\section{Conflict of Interest}

Authors stated no financial, personal, or professional conflicts of interest regarding this manuscript.

\section{Sources of Support:}

This work was supported by National Institutes of Health grants NS039123 to W. M. B. and HD02528 to the University of Kansas. The Hoglund Brain Imaging Center is supported by C76 HF00201 and a grant from Forrest and Sally Hoglund. W. M. B. is also supported by HD050534, AG029615, AG026374, AG026482, DK080090, and RR015563. C. M. C. is supported by the American Heart Association.

\section{Corresponding Author:}

Sorin C. Craciunas M. D. , Ph. D.

Staff Neurosurgeon, Neurosurgery Unit IV, Bagdasar-Arseni Hospital, 10-12, Berceni Avenue, Bucharest, Romania e-mail: craciunassorin@yahoo.fr

\section{References}

1. Agha A, Thompson CJ: Anterior pituitary dysfunction following traumatic brain injury (TBI). Clin Endocrinol (Oxf) 64:481-488, 2006.

2. Aimaretti G, Ambrosio MR, Di Somma C, Fusco A, Cannavo S, Gasperi M, Scaroni C, De Marinis L, Benvenga S, degli Uberti EC, Lombardi G, Mantero F, Martino E, Giordano G, Ghigo E: Traumatic brain injury and subarachnoid haemorrhage are conditions at high risk for hypopituitarism: screening study at 3 months after the brain injury. Clin Endocrinol (Oxf) 61:320-326, 2004.

3. Aimaretti G, Ghigo E: Traumatic brain injury and hypopituitarism. ScientificWorldJournal 5:777-781, 2005.

4. Benvenga S, Campenni A, Ruggeri RM, Trimarchi F: Clinical review 113: Hypopituitarism secondary to head trauma. J Clin Endocrinol Metab 85:1353-1361, 2000 .

5. Bondanelli M, De Marinis L, Ambrosio MR, Monesi M, Valle D, Zatelli MC, Fusco A, Bianchi A, Farneti M, degli Uberti EC: Occurrence of pituitary dysfunction following traumatic brain injury. J Neurotrauma 21:685-696, 2004.

6. Brooks WM, Friedman SD, Stidley CA: Reproducibility of $1 \mathrm{H}-\mathrm{MRS}$ in vivo. Magn Reson Med 41:193-197, 1999.

7. Cyran E: Pituitary damage due to basal skull fracture (in German). Deutsche Medizinische Wochenschrift 44:1261, 1918.

8. Dusick JR, Wang C, Cohan P, Swerdloff R, Kelly DF: Chapter 1: pathophysiology of hypopituitarism in the setting of brain injury. Pituitary, 2008.

9. Estes SM, Urban RJ: Hormonal replacement in patients with brain injury-induced hypopituitarism: who, when and how to treat? Pituitary 8:267-270, 2005.

10. Finkelstein E CP, Miller $T$ and associates: The Incidence and Economic Burden of Injuries in the United States. New York (NY): Oxford University Press; , 2006.

11. Giordano G, Aimaretti G, Ghigo E: Variations of pituitary function over time after brain injuries: the lesson from a prospective study. Pituitary 8:227-231, 2005.

12. Kelly DF, Gonzalo IT, Cohan P, Berman N, Swerdloff R, Wang C: Hypopituitarism following traumatic brain injury and aneurysmal subarachnoid hemorrhage: a preliminary report. J Neurosurg 93:743752, 2000

13. Klose M, Feldt-Rasmussen U: Does the type and severity of brain injury predict hypothalamo-pituitary dysfunction? Does post-traumatic hypopituitarism predict worse outcome? Pituitary, 2008.

14. Klose M, Juul A, Poulsgaard L, Kosteljanetz M, Brennum J, Feldt-Rasmussen U: Prevalence and predictive factors of post-traumatic hypopituitarism. Clin Endocrinol (Oxf) 67:193-201, 2007.

15. Klose M, Juul A, Struck J, Morgenthaler NG, Kosteljanetz M, Feldt-Rasmussen U: Acute and longterm pituitary insufficiency in traumatic brain injury: a prospective single-centre study. Clin Endocrinol (Oxf) 67:598-606, 2007.

16. Kreitschmann-Andermahr I: Subarachnoid 
hemorrhage as a cause of hypopituitarism. Pituitary 8:219-225, 2005.

17. Langlois JA R-BW, Thomas KE. : Traumatic brain injury in the United States: emergency department visits, hospitalizations, and deaths. Centers for Disease Control and Prevention, Nation Center for Injury Prevention and Control, 2006.

18. Lieberman SA, Oberoi AL, Gilkison CR, Masel BE, Urban RJ: Prevalence of neuroendocrine dysfunction in patients recovering from traumatic brain injury. J Clin Endocrinol Metab 86:2752-2756, 2001.

19. Lorenzo M, Peino R, Castro AI, Lage M, Popovic V, Dieguez C, Casanueva FF: Hypopituitarism and growth hormone deficiency in adult subjects after traumatic brain injury: who and when to test. Pituitary 8:233-237, 2005.

20. Maiya B, Newcombe V, Nortje J, Bradley $P$, Bernard F, Chatfield D, Outtrim J, Hutchinson P, Matta B, Antoun N, Menon D: Magnetic resonance imaging changes in the pituitary gland following acute traumatic brain injury. Intensive Care Med 34:468-475, 2008.

21. Masel BE: Traumatic brain injury induced hypopituitarism: the need and hope of rehabilitation. Pituitary 8:263-266, 2005.

22. Moeller TB, Reiff E: Pocket atlas of sectional anatomy: Head and Neck. Thieme, 2007.

23. Popovic V, Aimaretti G, Casanueva FF, Ghigo E: Hypopituitarism following traumatic brain injury. Growth Horm IGF Res 15:177-184, 2005.

24. Powner DJ, Boccalandro C, Alp MS, Vollmer DG: Endocrine failure after traumatic brain injury in adults. Neurocrit Care 5:61-70, 2006.

25. Salehi F, Kovacs K, Scheithauer BW, Pfeifer EA, Cusimano M: Histologic study of the human pituitary gland in acute traumatic brain injury. Brain Inj 21:651656, 2007.
26. Samadani U, Reyes-Moreno I, Buchfelder M: Endocrine dysfunction following traumatic brain injury: mechanisms, pathophysiology and clinical correlations. Acta Neurochir Suppl 93:121-125, 2005.

27. Schneider HJ, Samann PG, Schneider M, Croce CG, Corneli G, Sievers C, Ghigo E, Stalla GK, Aimaretti G: Pituitary imaging abnormalities in patients with and without hypopituitarism after traumatic brain injury. J Endocrinol Invest 30:RC9-RC12, 2007.

28. Schneider M, Schneider HJ, Yassouridis A, Saller B, von Rosen F, Stalla GK: Predictors of anterior pituitary insufficiency after traumatic brain injury. Clin Endocrinol (Oxf) 68:206-212, 2008.

29. Tanriverdi F, De Bellis A, Bizzarro A, Sinisi AA, Bellastella G, Pane E, Bellastella A, Unluhizarci K, Selcuklu A, Casanueva FF, Kelestimur F: Antipituitary antibodies after traumatic brain injury: is head traumainduced pituitary dysfunction associated with autoimmunity? Eur J Endocrinol 159:7-13, 2008.

30. Tanriverdi F, Senyurek H, Unluhizarci K, Selcuklu A, Casanueva FF, Kelestimur F: High risk of hypopituitarism after traumatic brain injury: a prospective investigation of anterior pituitary function in the acute phase and 12 months after trauma. J Clin Endocrinol Metab 91:2105-2111, 2006.

31. Tanriverdi F, Ulutabanca H, Unluhizarci K, Selcuklu A, Casanueva FF, Kelestimur F: Three years prospective investigation of anterior pituitary function after traumatic brain injury: a pilot study. Clin Endocrinol (Oxf) 68:573-579, 2008.

32. Teasdale G, Jennett B: Assessment of coma and impaired consciousness. A practical scale. Lancet 2:8184, 1974.

33. Thurman DJ, Alverson C, Dunn KA, Guerrero J, Sniezek JE: Traumatic brain injury in the United States: A public health perspective. J Head Trauma Rehabil 14:602-615, 1999. 